
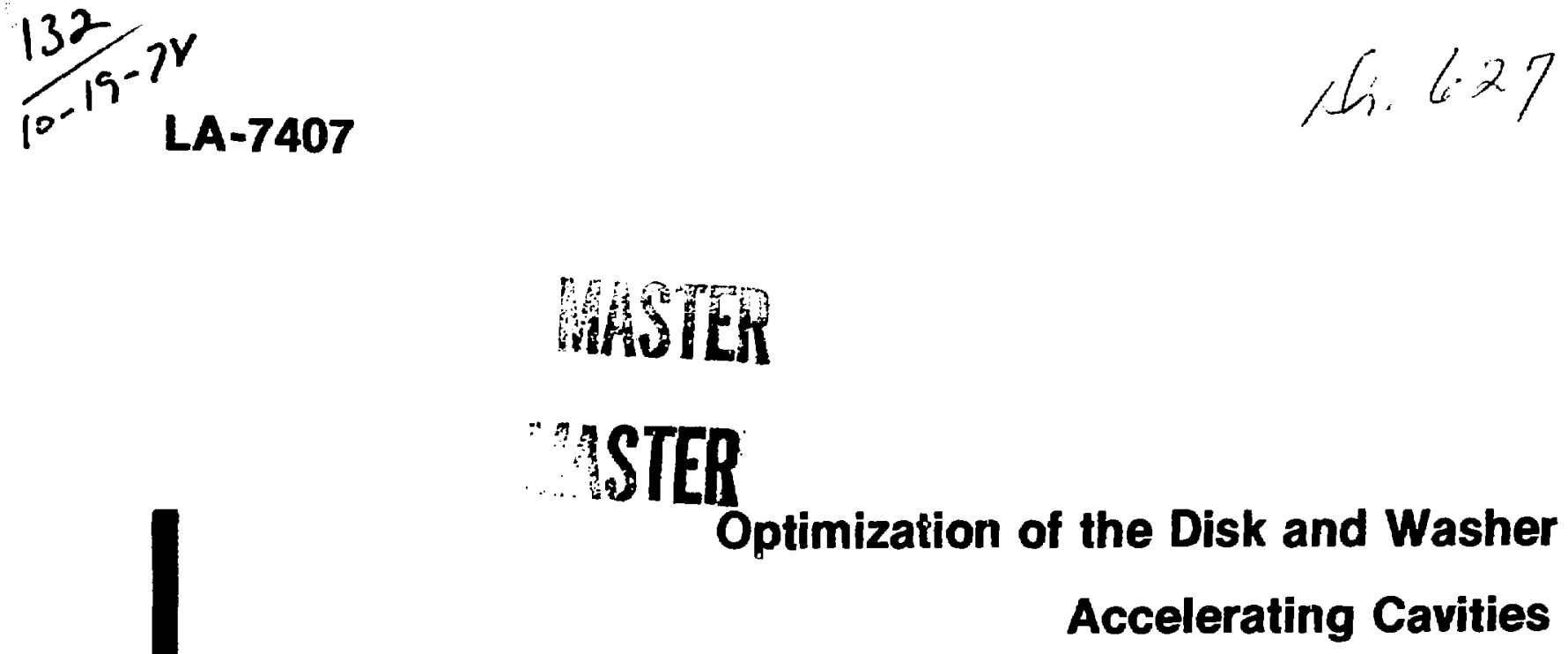

$\frac{\pi}{5}$
$\frac{0}{0}$
$\frac{0}{0}$
$\frac{1}{0}$
$\frac{2}{0}$
$\frac{5}{5}$ 


\title{
Optimization of the Disk and Washer Accelerating Cavities
}

\author{
Joseph J. Manca \\ Edward A. Knapp
}

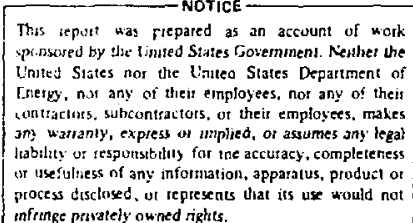




\title{
OPTIMIZATION OF THE DISK AND WASHER ACCELERATING CAVITIES
}

\author{
by
}

\author{
Joseph .J. Manca and Edward A. Knapp
}

\begin{abstract}
High-energy accelerator structures should have not onIy a high shunt impedance, $Z$, by which the $r f$ power can be used efficiently for particle acceleration, but also a high coupling, $k$, between the neighboring cells. A study was made of the disk and washer (DAW) accelerating structure. The DAW structure has high coupling $(k \sim 50 \%)$ as well as a high shunt impedance comparable to that of a side-coupled structure and can be used successfully in high-current accelerators. Two DAW configurations were studied: DAW1, which has a flat washer in the central region, and DAW2, in which the upper part of the washer is curved. The latter design does not derrease the effective shunt impedance of the structure but does permit a smallor cavity diameter (principally for higher $\beta$ ) than does the straightwasher lesign. The two structures were optimized by the SUPERFISH computer sode.
\end{abstract}

\section{INTRODUCTION}

In general, high-energy proton linear accelerators consist of two main sections, a drift-tube-type structure that accelerates the protons to approximately half the speed of light $(150 \mathrm{MeV})$ and a coupledcavity section that continues the acceleration to the final output energy. Here we discuss the coupledcavity section, which must have both a high efficiency to convert rf power into particle energy and a stable operating mode to allow precise field control under beam-loaded conditions. In particular, we analyze the disk and washer (DAW) structure for shunt-impedance optima and tuning criteria. Characteristics of the coupled-cavity mode will be discussed in a future report. The DAW structure has a high efficiency, excellent tuning characteristics, and can serve as a new, tightly coupled accelerator structure suitable for use in high-energy accelerators.

\section{DISK AND WASHER STRUCTURE}

The DAW structure was invented by V. G. Andreev of the Radiotechnical Institute in Moscow. ${ }^{1-3}$ The basic configuration of the DAW structure with the straight washer (DAW1) is shown in Fig. 1. The washer is the part with the on-axis cones resembling drift tubes; the disk is on the circumference of the cavity between the two washers and is similar to an iris of an iris-loaded waveguide.

The DAW structure has the following peculiarities.

1) $\mathrm{A} T \mathrm{TM}_{02}$-like mode is excited in the cavity as an accelerating mode at $\pi / 2$ (accelerating cells of 


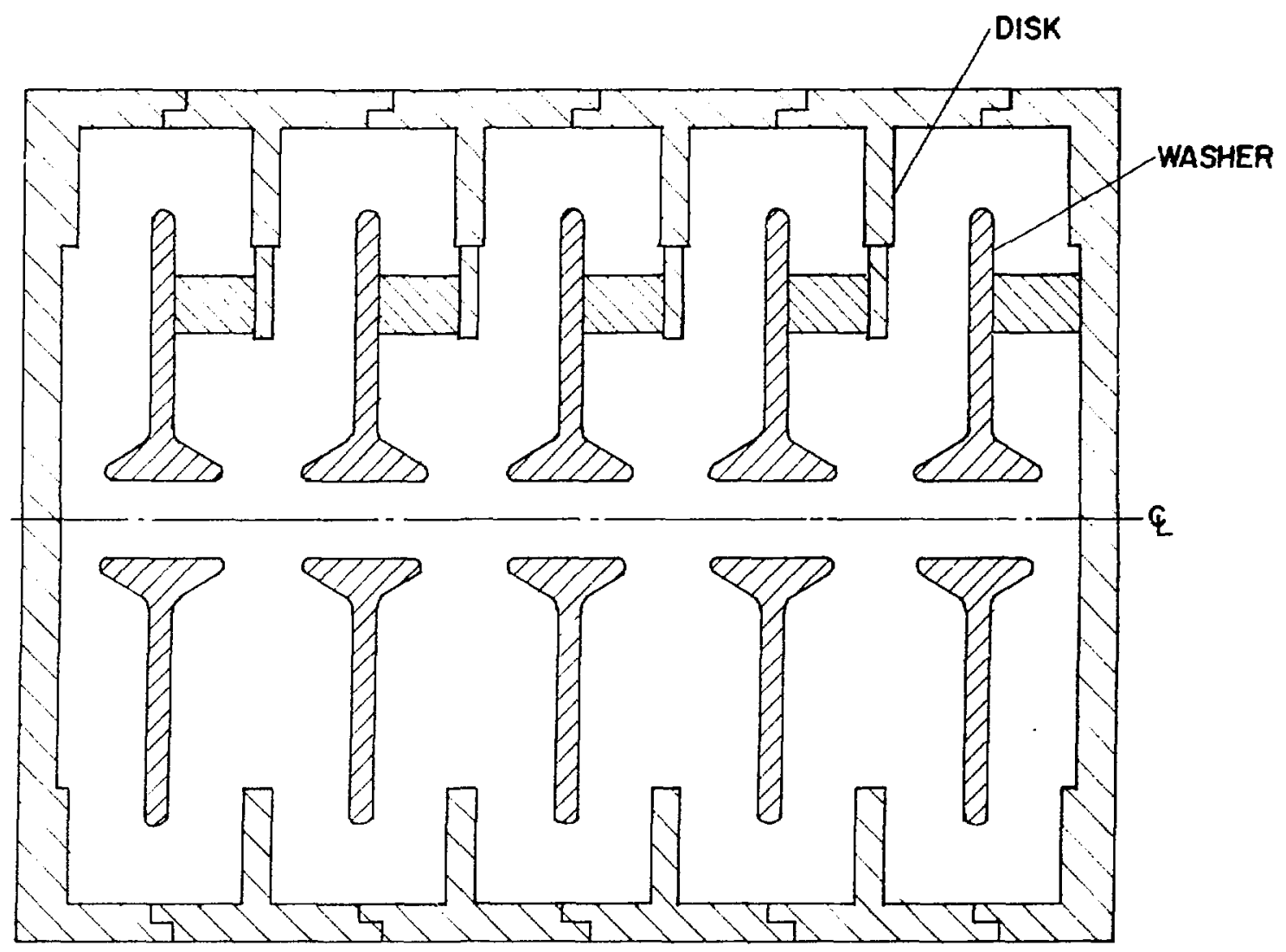

Fig. 1.

Disk and washer (DAW) structure.

side- or ring-coupled structures are excited in the $\mathrm{TM}_{\mathbf{0 1}}$ mode).

2) No obvious separation exists between the accelerating and coupling cavities. Excitation of the accelere: ing or coupling $\pi / 2$ mode depends on the bounilay conditions. When a cavity is shaped as in Fig. 2a, with the conducting plates at both ends which correspond to the Neumann type- $\mathrm{N}$ boundaries, the accelerating mode (with the field lines shown) is supported. When a cavity like that in Fig. $2 b$ is formed. a coupling mode (with the field lines shown) is excited.

3) High coupling between the accelerating and coupling modes results from the strong overlapping of the electromagnetic fields of the two modes; consequently, a wide passband is possible when the two $\pi / 2$ modes are of the same frequency.
4) A cavity such as that shown in Fig. 2 has two additional modes: $\mathrm{TM}_{01}$ and $\mathrm{TM}_{02}$, which belong to the 0 and $\pi$ modes of the dispersion curve, respectively (Figs. 2c and d). When more cavities are used for a structure, all additional modes of the dispersion curve lie between these two modes.

5) Clearly, the cavity of Fig. 2 cannot be divided into individual accelerating and coupling cells. For optimization and tuning purposes, the cavity must be taken as a unit - a module consisting of one washer and one disk (see Fig. 3).

6) A low If current characterizes the outer wall of the cavity where the joint is made between the modules. Therefore, the imperfections in the joint do not contribute strongly to the field perturbations and rf losses in the cavity. 


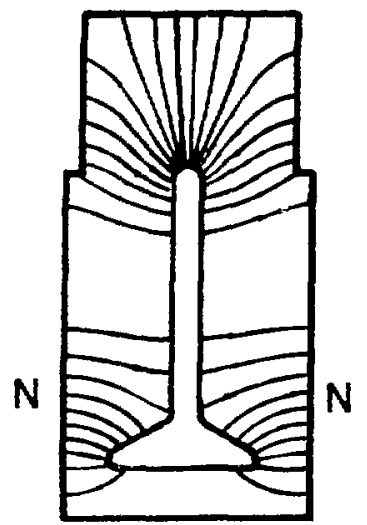

a) $\pi / 2$ accelerating mode

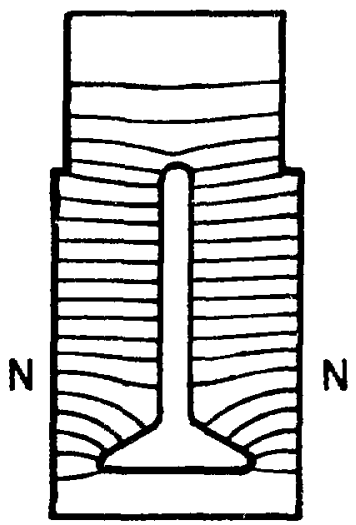

c) $T M_{\text {OI }}$ mode

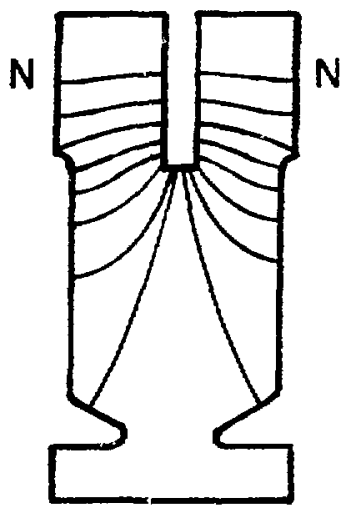

b) $\pi / 2$ coupling mode

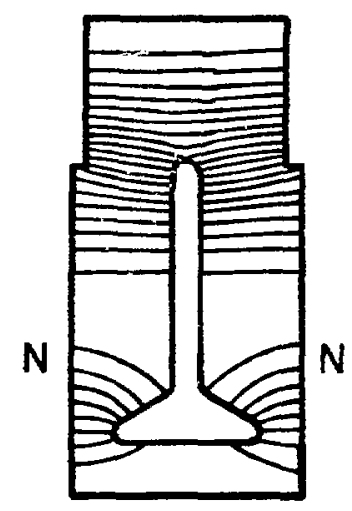

d) $\mathrm{TM}_{\mathrm{O} 2}$ mode

Fig. 2.

$D A W$ cavity modes. a) $\pi / 2$ accelerating $\left(\mathrm{TM}_{02}\right.$-like) mode. b) $\pi / 2$ coupling mode. c) " $\mathrm{O}$ " $\left(\mathrm{TM}_{01}\right)$ mode. $\left.\mathrm{d}\right) " \pi "\left(\mathrm{TM}_{02}\right)$ mode. 


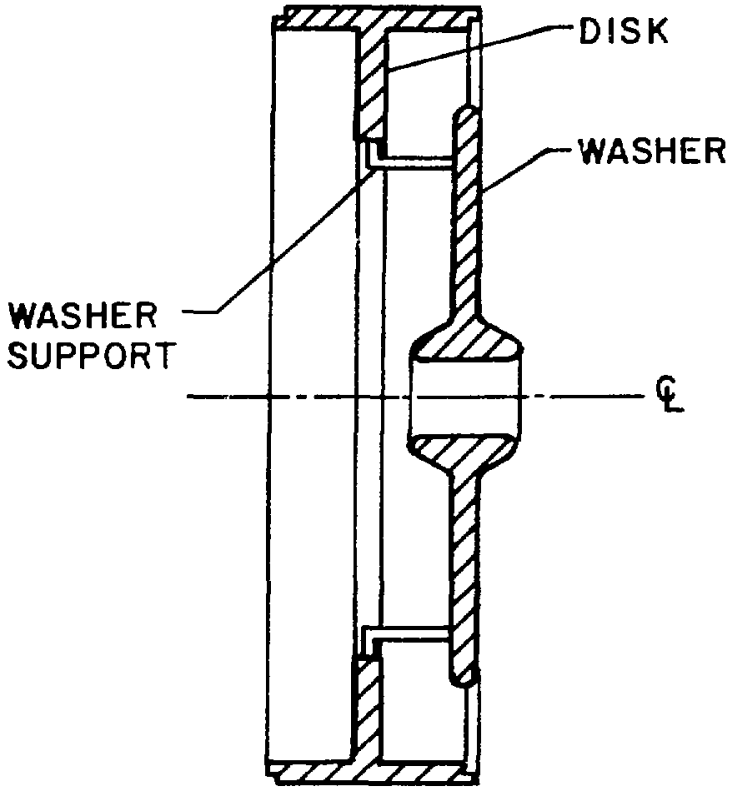

Fig. 3.

DA $W$ module.

Figure 2 shows that the field distribution in the cavity is symmetrical about the cavity's middle plane. For that reason, one half-cavity is sufficient for the calculations, and the results then can be applied to the whole cavity. Figure 4 shows the basic lualf-cavity and the notations of the geometrical parameters used in our calculations. When such a half-cavity is terminated by all-metallic, Neumann type- $\mathrm{N}$ boundaries, two modes $\left(\mathrm{TM}_{01}\right.$ and $\mathrm{TM}_{02}$ ) should be calcuiated (see Figs. 5a and b). When the metallic boundary is used on the left side of the cavity but the right side has a nonmetallic, Dirichlet type-D boundary, the accelerating mode should be calculated (Fig. $5 \mathrm{c}$ ). When the boundary types are reversed, a coupling mode should be calculated (Fig. $5 \mathrm{~d}$ ). Fields in the cavity were calculated by the SUPERFISH program. ${ }^{4}$

In our calculations, the ideal cavity has a floating washer-an impractical arrangement. In practice, stems to support the washer are required at locations where they least perturb the cavity fields. The field distributions in Figs. $2 b$ and $d$ indicate that the electric field changes its sign in the cases of the accelerating mode and the true $\mathrm{TM}_{02}$ mode, and a region of zero electric field exists. Washer-support stems located in this region would least perturb the

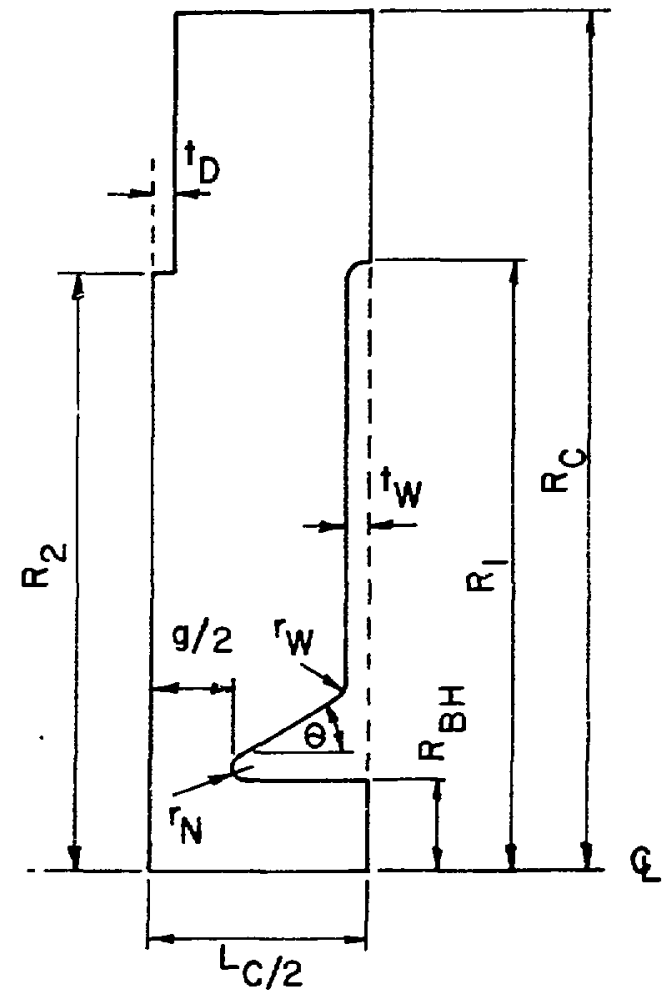

Fig. 4.

Geometrical parameters of DAW1.

cavity field. The coupling mode (Fig. $2 \mathrm{c}$ ) has a weak electric field in the region of the stem supports, and strong field perturbation should not be expected.

The most perturbed mode is the $T M_{01}$ because the metallic stems make a short circuit in the electric field. These perturbations do not seriously affect the structure's properties but must be taken into account during experimental tuning. Although the stems cause field perturbations and increased power losses in the cavity, they can be used successfully to transport cooling fluid into the washer, where the significant part of the total power is dissipated. This possibility must be emphasized because it makes the DAW structure particularly suitable for highpower rf operation.

\section{GEOMETRY OF THE DAW1 CAVITY}

Because of the axial and middle plane symmetries, we use only one quadrant of cavity in our 


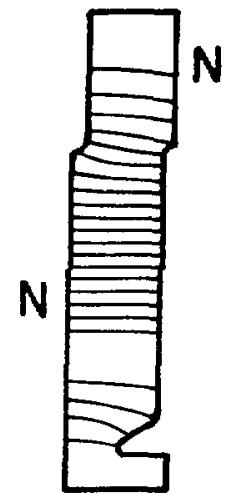

$t_{0}=735 \mathrm{MHz}$

a

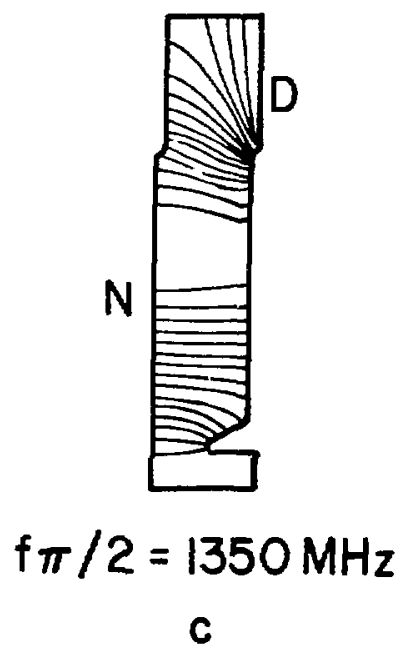

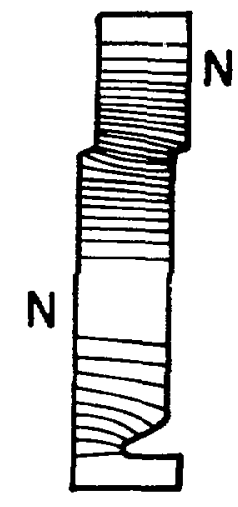

$f \pi=1631 \mathrm{MHz}$

b

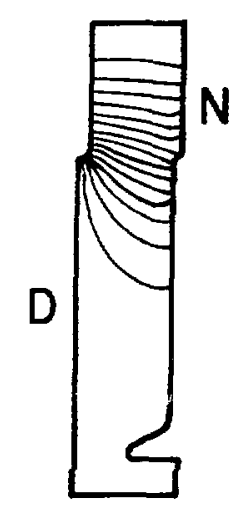

$f \pi / 2=1350 \mathrm{MHz}$

d

Fig. 5.

Half-cavity modes.

calculations, as we did in the case of the $\mathrm{TM}_{01}$ cavity. ${ }^{5}$ Geometrical parameters are indicated in Fig. 4 and described below.

$R_{c}=$ cavity radius

$\mathrm{L}_{\mathrm{c}}=$ cavity length $(\mathrm{L}=\beta \lambda / 2)$

$\beta=$ relative particle velocity

$\lambda=$ resonant frequency wavelength

$\mathrm{g}=$ gap length $\left(\mathrm{g}=\alpha \mathrm{L}_{\mathrm{c}}\right)$ $x=$ gap factor

$r_{B H}=$ bore-bole radius

$0=$ cone angle

$\mathrm{r}_{\mathrm{v}}=$ cone radius

$t_{w}=$ half-washer thickness

$t_{D}=$ half-disk thickness

$R_{1}=$ washer radius

$R_{2}=$ inner radius of disk. 


\section{PHYSICAL PARAMETERS}

Definitions of the DAWl's physical parameters are given in the literature (for example, Ref. 6), as are descriptions of the SLPERFISH calculation of the parameters (Ref. 5). Reference 4 contains more information on SUPERFISH.

Results for the calculations described here are normalized to $\mathrm{E}_{z_{0}}=1 \mathrm{WV} / \mathrm{m}$ and the calculations were made at $f_{r}=1350 \mathrm{MHz}$.

\section{OPTIMIZATION PROCEDURE}

Figure 4 show's that there are many geometrical parameters on which the electrical properties of the cavity depend. Moreover, there are two frequencies $\left(f_{2}\right.$ and $\left.f_{3}\right)$ corresponding to the accelerating and coupling modes, respectively, instead of the one frequency found in the $T \mathrm{M}_{01}$ cavity. The two frequencies must be equal $\left(f_{2}=f_{3}\right)$ if a closed dispersion curve is to be achieved at the $\pi / 2$ mode. In addition. two more frequencies $\left(f_{0}\right.$ and $f_{4}$ ) correspond to the $\mathrm{TM}_{0,1}$ and $\mathrm{TM}_{02}$ modes. Evidently the change of any yeometrical parameter affects all frequencies, but in different ways.

In the optimization procedure one looks for the highest possible effective shunt impedance, $Z T^{2}$, by determining optimum cavity geometry. To optimize the DAW cavity with respect to all geometrical parameters would be extremely difficult and timeconsuming (mainly expensive computing time). Fortunately, we found for the optimized DAW structure that the region of the cavity close to the axis is similar to the equivalent region in the optimized $T M_{01}$ cavity used, for example, in the sidecoupled structure. The electrical properties (frequency, shunt impedance) of the two cavities vary similarly with close-to-axis geometrical parameter changes. Consequently, we optimized the $\mathbf{T M}_{01}$ cavity ${ }^{5}$ and applied the results to the DAW cavity. $\mathrm{Ni}$ then studied DAW cavity behavior by changing only a few parameters connected to the outer part of the DAW cavity:

$$
\begin{aligned}
& 2 \mathrm{R}_{1}=\text { washer diameter } \\
& 2 \mathrm{R}_{2}=\text { inner diameter of disk } \\
& 2 \mathrm{R}_{c}=\text { cavity diameter } \\
& 2 \mathrm{t}_{\mathrm{D}}=\text { disk thickness } \\
& 2 \mathrm{t}_{w}=\text { washer thickness. }
\end{aligned}
$$

Washer thickness has practically the same efiect on the physical parameters of the DAW cavity that it has on the $T \mathrm{M}_{01}$ accelerating cavity. In addirion. one must consider the effect of washer thickness on all frequency changes, even those that are not drastic. In spite of the desirability of obtaining a higher $Z T^{2}$ by making $2 t_{w}$ small, the mechanical constraints of possible water cooling must be kept in mind when determining washer thickness.

Effective shunt impedance $\mathrm{ZT}^{2}$ does not change significantly with disk thickness $2 t_{1}$. and mechanical constraints can be applied to determine the correct disk thickness.

After the near-axis cavity garameters and washer and disk thicknesses have been established, washer diameter and the inner diameter of the disk must be calculated so that the frequency $f_{2}=f_{r}$. In our case. $\mathrm{f}_{\mathrm{r}}=1.50 \mathrm{MHz}$ - the operating frequency of the high-energy section of the proton linear accelerator designed for hospital-based pion generation being investigated at the Los Alamos Scipntific Laboratory.

The parameters $R_{1}$ and $R_{2}$ must then be adjusted so that the frequency $f_{2}$ equals the design frequency $f_{\mathrm{r}}$. Both parameters can be calculated analytically. as was done by Andreev, but we found it more conrenient to use SLPERFISH.

The other $\pi / 2$ mode frequency $\left(f_{3}\right)$ must equal $f_{2}$. a requirement that at this point can be fulfilled only by adjusting the cavity diameter $2 R_{c}$.

\section{OPTIMIZATION RESULTS FOR DAWI}

The variation of geometrical parameters affects the resonant frequencies and the physical parameters in a way that can be calculated either analytically or by computer code. Use of a computer program can be expedited if one knows the effert of cavity frequency changes on the changes of the geometrical parameters before optimizing a particular physical parameter. For DAW1, Table I shows the directions of the frequency changes with respect to the specific parameter and the approximate rate of changes as calculated by SUPERFISH.

We used the optimized geometrical parameters of the $\mathrm{TM}_{01}$ cavity for the near-axis region of the DAW1 cavity because the general behavior of the physical parameters of the two are similar. Also, all 


\section{TABLE I}

\section{DIRECTIONS AND RATES OF FREQLENCY CHANGES WITH PARAMETER CHANGES FOR DAW 1}

\begin{tabular}{|c|c|c|c|c|}
\hline Parameter & $\mathbf{f}_{2}$ & $\begin{array}{c}\text { Rrite } \\
(\mathrm{MH} / \mathrm{cm})\end{array}$ & $\mathbf{f}_{3}$ & $\begin{array}{c}\text { Rate } \\
(\mathrm{MHz} / \mathrm{cm})\end{array}$ \\
\hline $\mathrm{R}^{*} \cdot$ & $:$ & 10 & : & 200 \\
\hline$R_{t}$ and $R_{2}$ & : & 100 & $\cdot$ & 190 \\
\hline$t_{w} \uparrow$ & • & 200 & ..- & -.. \\
\hline$t_{1} \cdot$ & -.- & $-\cdot$ & $\cdot$ & 101 \\
\hline $\mathrm{g}:$ & $\cdot$ & 300 & $\ldots$ & -.. \\
\hline
\end{tabular}

the considerations made for the $T M_{01}$ cavity with respect to these near-axis parameters can be applied to the DAWl cavity.

We assume:

$$
\begin{aligned}
& \mathrm{f}_{r}=1.350 \mathrm{MHz} \\
& r_{\mathrm{B} U}=1.1 \mathrm{~cm} \\
& r_{N}=0.25 \mathrm{~cm} \\
& 0=30^{\circ} \\
& \mathrm{t}_{w}=0.35 \mathrm{~cm} \\
& r_{w}=0 \mathrm{~cm} .
\end{aligned}
$$

The above parameters remain constant for all values of $\beta$.

The dependence of the optimum gap factor on the relative particle velocity is shown in Fig. 6 . The value of $\alpha_{o n}$ does not change with any parameter other than bore-hole radius $\mathrm{I}_{\mathrm{H} H}$. Disk thickness $2 \mathrm{t}_{\mathrm{D}}$ affects mainly frequeney $f_{3}$ and has little effect on frequency $f_{2}$. The effective shunt impedance $Z \mathrm{~T}^{2}$ in. creases slightly with disk thickness: 2 to $3 \%$ in the range $2 t_{\mathrm{p}}=0.1$ to $\mathrm{L}_{\mathrm{c}} / 4$. If $2 \mathrm{t}_{\mathrm{p}}$ is made thicker to obtain a higher $2 T^{2}$, the effect of in on the dispersion curve's symmetry is undesirable. Therefore. $t_{n}$ should be so chosen that it fulfills mechanical requirements.

The washer radius $R_{1}$ and the inner radius of the disk $R_{2}$ must then be fixed to satisfy the condition $f_{2}=f_{r}$. The dependence of $R_{1}$ in relative particle velocity is shown in Fig. 7 .

The effective shunt impedance $\mathrm{ZT}^{2}$ rises when the inner radius of the disk $R_{2}$ is made larger than $R_{3}$.

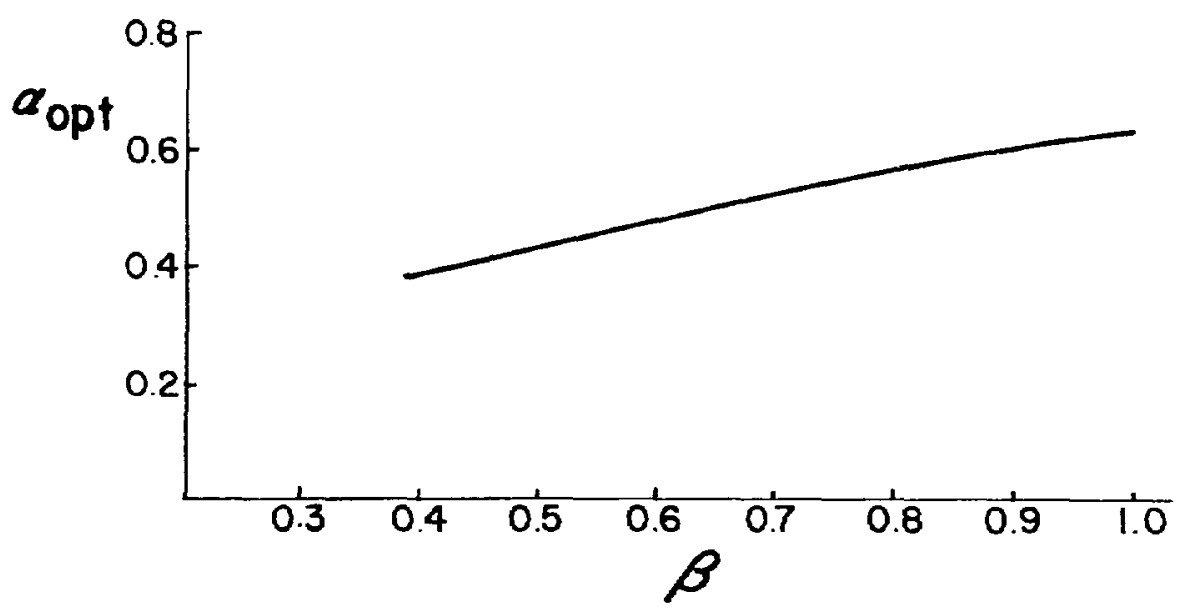

Fig. 6 .

Optimum gap factor $\alpha_{\mathrm{op}}$ as a function of relative particle velocity 3 . 


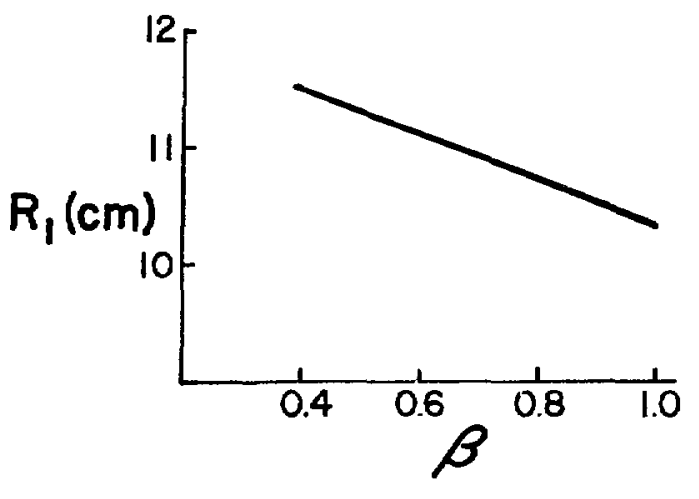

Fig. 7.

Washer radius $R_{1}$ as a function of $\beta$.

but increasing $R_{2}$ above $R_{1}$ leads to asymmetry of the dispersion curve. A compromise in which $R_{2}$ is 2 to $3 \%$ smaller than $R_{1}$ gives the best results. Cavity radius $R_{c}$ has little effect on the accelerating-mode parameters, but does have a determining effect on the coupling-mode frequency $\mathrm{f}_{\mathrm{s}}$. The calculated change of $f_{3}$ on $R_{c}$ is shown in Table $I$. This last parameter is used to equalize frequencies $f_{2}=f_{3}$. Our best results were obtained with the ratio $\mathrm{R}_{1} / \mathbf{R}_{c}$ $=0.7$ to 0.75 . Table I also shows the effect of other parameters on $f_{2}$ and $f_{3}$ frequencies. Figure 8 shows the dependence of cavity radius $R_{c}$ on relative particle velocity $\beta$.

\section{RF POWER DISSIPATION IN THE DAW] CAVITY}

The major part of the rf power fed into the DAW1 cavity is lost in the washer. In a practical design, the washer must be cooled (four washer-support stems with holes for cooling-liquid transport could be used). Figure 9 shows a typical power-loss distribution on the cavity walls. The ratio of power loss in the washer to the total power loss in the cavity changes with relative particle velocity $\beta$. As shown in Fig. 10, over $90 \%$ is lost in the washer at $\beta=0.4$ and less than $60 \%$ is lost at $\beta=1.0$.

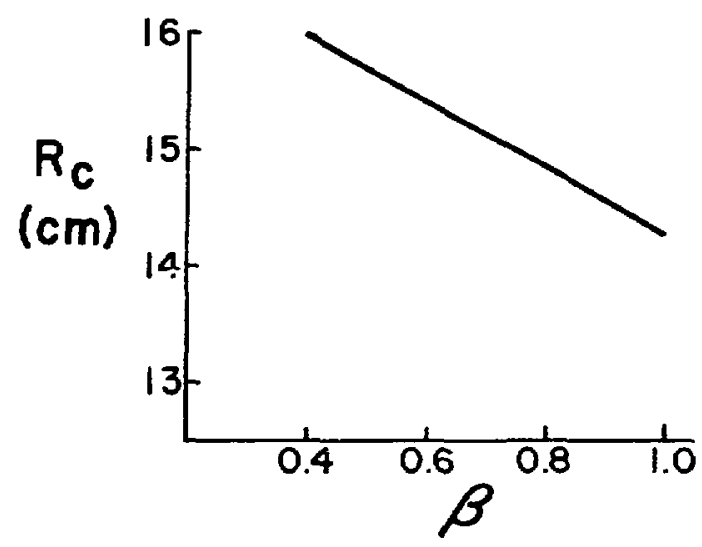

Fig. 8.

Cavity radius $R_{\mathrm{c}}$ as a function of $\beta$.

\section{DAW2 CAVITY}

As we discovered during our DAW1 optimization. interesting things happen when the upper part of the washer is curved and a second nose is made at the outer part of the washer.

1) Both accelerating- and coupling-mode frequencies $\left(f_{2}\right.$ and $\left.f_{3}\right)$ are lowered. To compensate for that and to bring frequencies up to $f_{2}=f_{3}=f_{r}$, the cavity radius $R_{c}$ and the washer radius and inner radius of the disk $\left(R_{2}\right.$ and $\left.R_{2}\right)$ must be diminished.

2) The effective shunt impedance $\mathrm{ZT}^{2}$ does not change. The second nose causes increased of losses in the cavity, but they are compensated for by the smaller cavity dimensions.

3) Quality factor $Q$ decreases about $15 \%$ with respect to DAW1 cavity $Q$, but it is still higher than the $Q$ of the single $T M_{01}$ cavity.

4) The electric-field distribution on the cavity axis is not affected and the transmit-time factor remains the same.

5) The electric field of the cavity's outer region concentrates around the second nose, moving the zero-field region of the accelerating mode farther from the cavity axis, thereby allowing better positioning of the washer-support stems. 

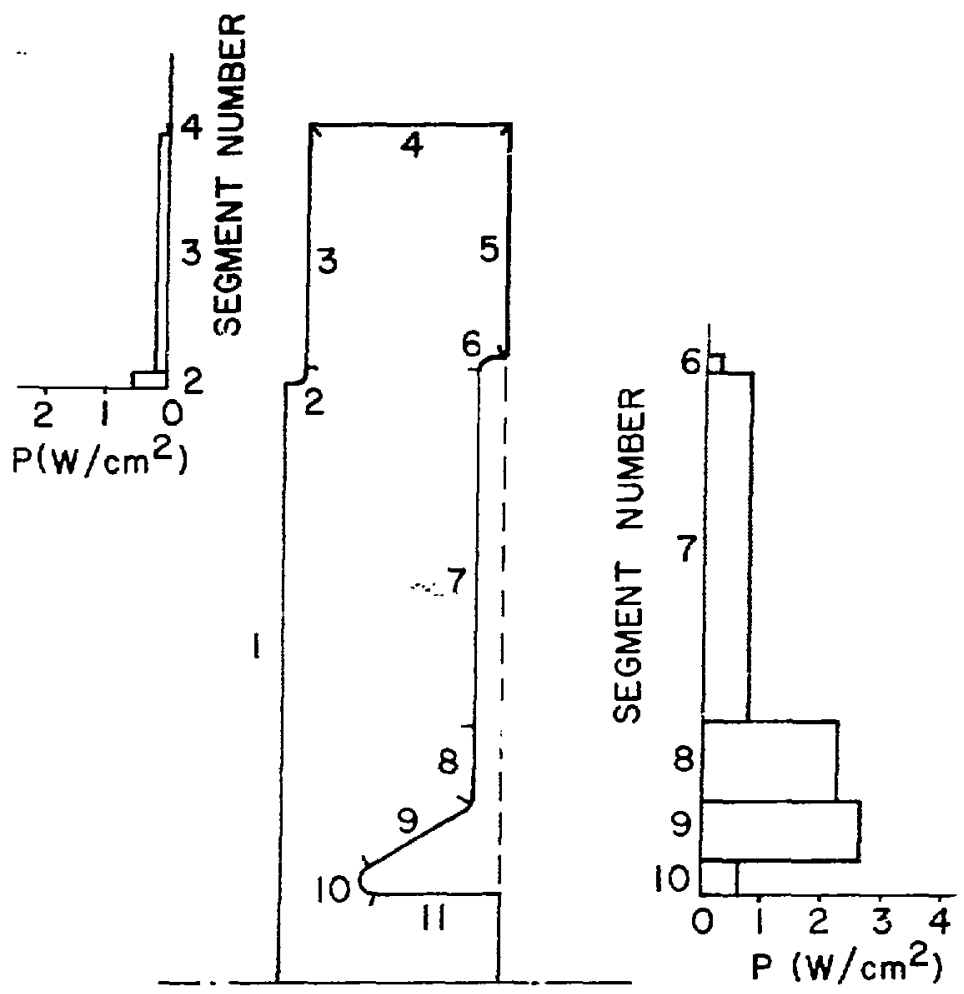

Fig. 9.

rf power-loss distribution on cavity ualls $(j=0.6)$.

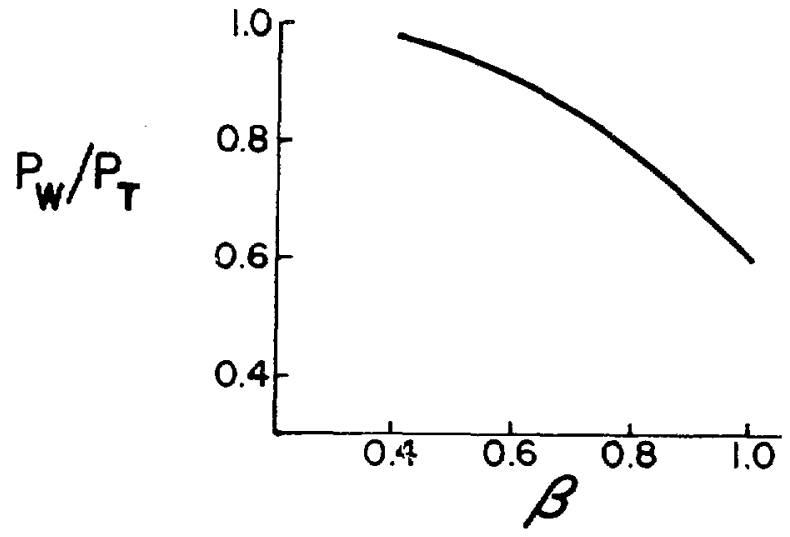

Fig. 10.

Ratio of rf power loss in washer to rf power loss in whole cavity as a function of $\beta$.

6) One suspects that the coupling of the transverse $\mathrm{TE}_{11}$ mode and its propagation in the structure would be attenuated so that for DAW2 the beam blowup current limit (caused by the excitation of the $T E_{1:}$ model may be higher than for DAWl.

Figure 11 shows the DAW2 cavity with the following geometrical parameters (in addition to DAW1 cavity parameters):

$\mathrm{R}_{\mathrm{w}}=$ washer wall radius

$r_{\mathrm{N}_{1}}=$ upper-gap nose radius

$r_{c}=$ upper-corner radius

$\mathrm{g}_{1}=$ upper-gap length

$\alpha_{1}=$ upper-gap factor $\left(a_{i}=g_{l} / L_{C}\right)$.

The upper-corner radius $r_{i}$ can be used for changing coupling-mode frequency $f_{3}$ in a small frequency range when the smallest possible change of accelerating-mode frequency $f_{2}$ is desired. When it is made a straight line, it can be useful in experimental cavity tuning.

Calculated modes for the $\beta=0.6$ half-DAW2 cavity are shown in Fig. 12. The effect of the individual geometrical parameter changes on DAH2 


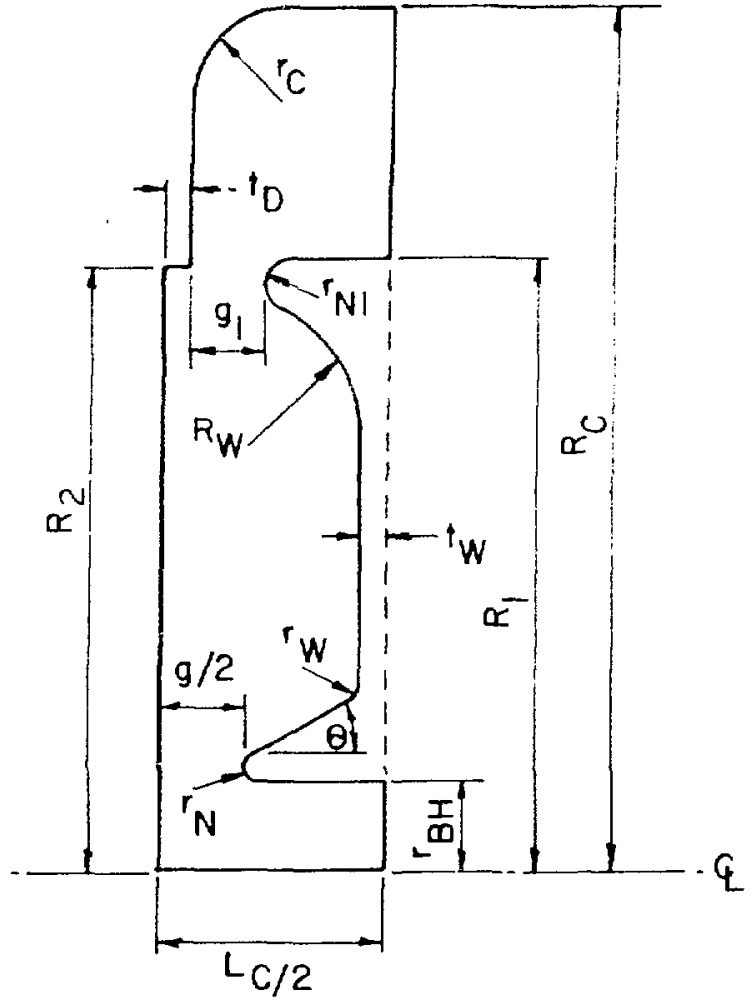

Fig. II.

IDA W2 geometr:

cavity trequencies and the approximate rates of frequency changes are shown in Table II.

\section{OPTIMIZATION RESULTS FOR THE DAW2 CAVI'TY}

The DAW2 cavity optimization procedure is the same as the one used for the DAWI cavity. In addition, to obtain a high $2 T^{2}$ we must optimize DAW2 parameters such as $\mathrm{R}_{\mathrm{w}}, \alpha_{1}$, and $\mathrm{r}_{\mathrm{N}_{1}}$. The best results are obtained when the washer wall radius is given by

$\mathrm{R}_{\mathrm{w}}=\mathrm{L}_{\mathrm{c}} / 2-\mathrm{t}_{\mathrm{w}}-\mathrm{t}_{\mathrm{p}}-\mathrm{g}_{1} / 2$

The effective shunt impedance changes slightly when the length $\left(g_{1}\right)$ changes. When $g_{1}$ is made too small the difficulty arises with the linearization of the dispersion curve and closing stop band around
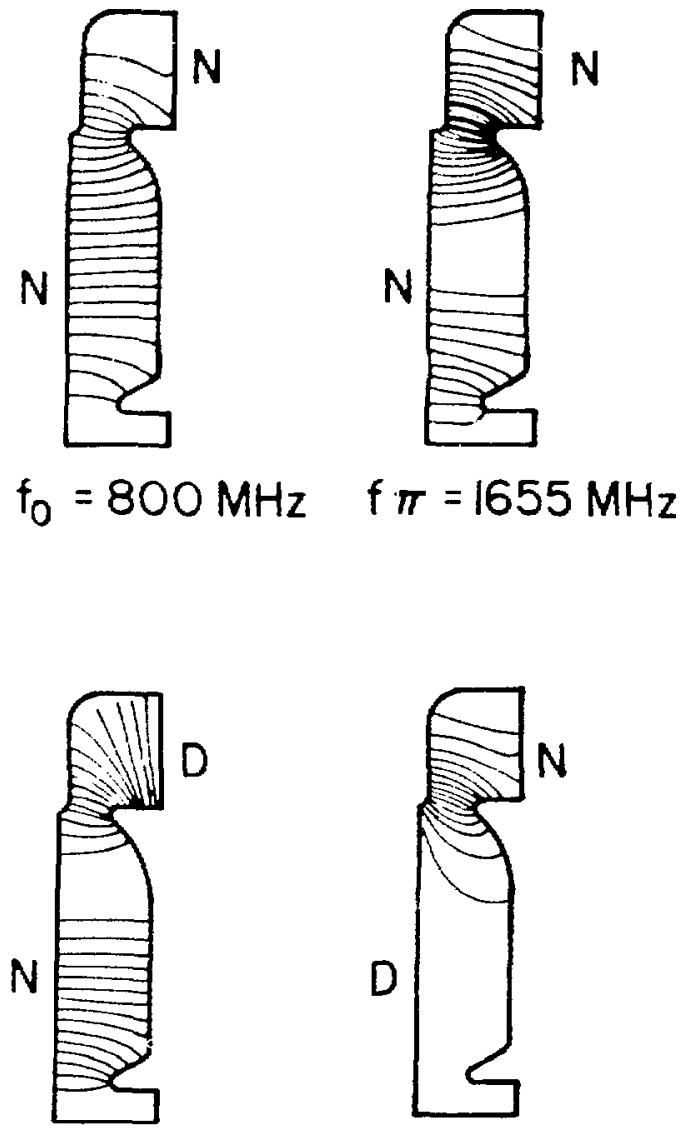

$f \pi / 2=1350 \mathrm{MHz} \quad f \pi / 2=1350 \mathrm{MHz}$

Fig. 12.

I) A W2 cacity modes.

the $\pi / 2$ mode. Therefore. the criteria for the dispersion curve must be taken into cronsideration when weighing the advantages of a second nose. The best results are obtained when the gap length is $g_{2} \geq L_{\alpha} / 4$ for all $\beta$.

Radius $r_{N}$, has a negligible effect on $Z T^{2}$ and the dispersion curve; consequently; its ralue is chosen so that it can withst and a maximum electric gradient on its surface. To avoid trouble connected with sparking, we choose $r_{N_{1}}=0.4$. The calculated gradient is always an order of magnitude less than the gradient on the lower-gap nose surface for all $\beta$.

With the geometrical parameters predetermined. we calculate the physical parameters of the DAW2 cavity. Effective shunt impedance $\mathrm{ZT}^{2}$ as a function 


\section{T:ABLE: II}

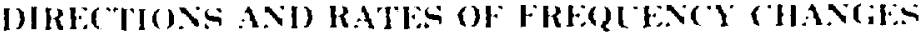

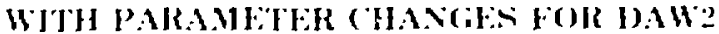

\begin{tabular}{|c|c|}
\hline & Rate \\
\hline
\end{tabular}

\begin{tabular}{|c|c|c|c|c|}
\hline$R \cdot$ & . & 111 & . & $2 ! 10$ \\
\hline$K$ and $K:$ & $\cdot$ & $1: 21$ & . & $\because 111$ \\
\hline$i_{n} \cdot$ & $\cdot$ & 3011 & $\ldots$ & \\
\hline $1:$ & $\ldots$ & $\cdots$ & . & $2 n+11$ \\
\hline$i^{2}$ & $\cdot$ & 2011 & $\ldots$ & 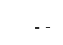 \\
\hline$\cdot$ & $\cdot$ & 111 & $\cdot$ & ins \\
\hline r. & $\ldots$ & $\ldots$ & . & (1) \\
\hline
\end{tabular}

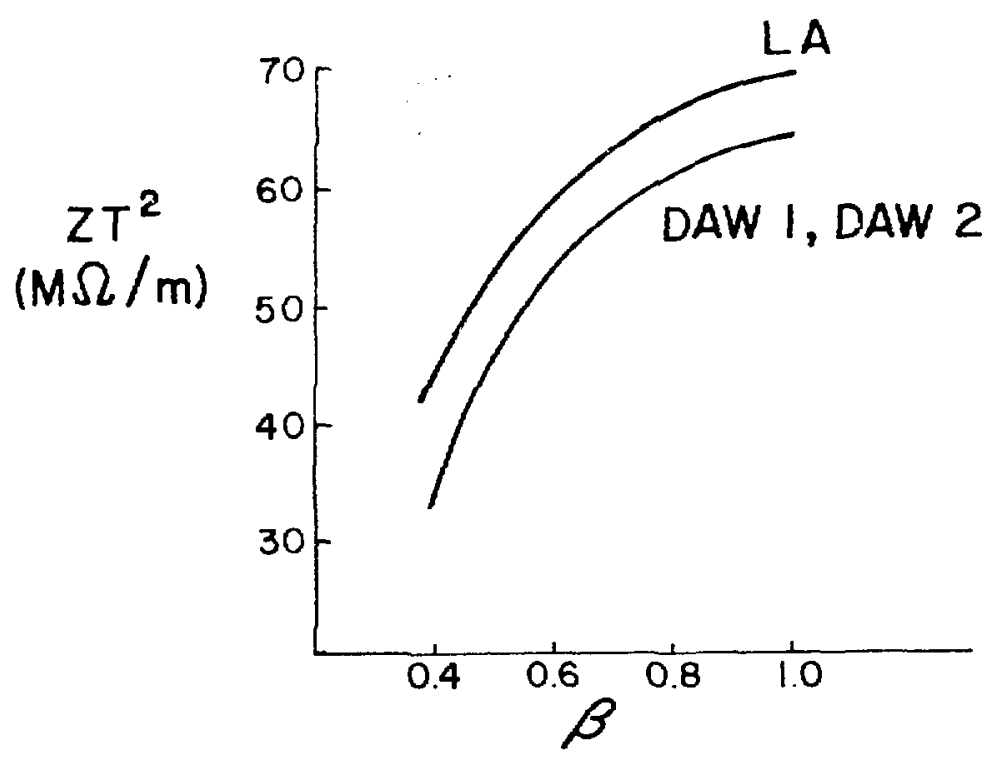

Fig. 13.

Effective shunt impedance $Z T^{2}$ as a function of $B$.

of $\beta$ is shown in Fig. 13. Other curves of the figure are for the DAW1 and $\mathrm{TM}_{01}$ cavities, and they illustrate the differences between the three most important structural configurations. Dependence of cavity radius $R_{c}$ on $\beta$ is shown in Fig. 14, where curves for the DAW1 and $\mathrm{TM}_{01}$ cavities again are plotted for comparison. The DAW2 cavity has a larger diameter than the $\mathrm{TM}_{01}$ cavity; however, its diameter is smaller than that of DAW1. A larger dif- ference betwen the two DAW diameters appears toward a higher $\beta$, where the difference between the DAW2 and $\mathrm{TM}_{01}$ cavities becomes smaller.

Washer radius $R_{2}$ as a function of $\beta$ is shown in Fig. 15. Quality factor $Q$ vs $\beta$ for the optimized DAW2, DAW1, and $\mathrm{TM}_{01}$ cavities is shown in Fig. 16. Energy stored in the cavity as a function of $\beta$ is shown in Fig. 17 for the three configurations. 


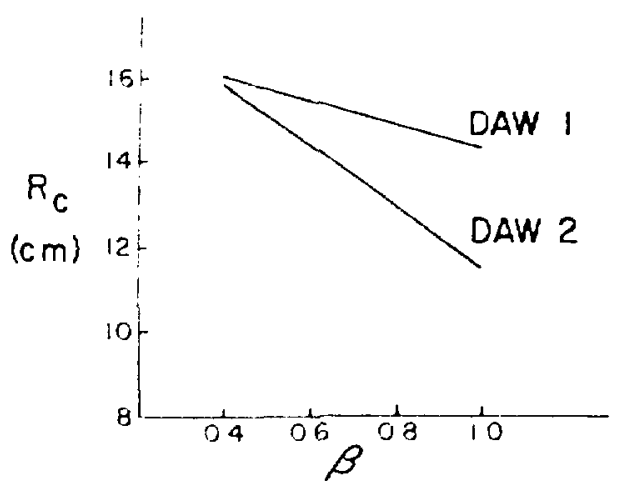

Fins. 1.

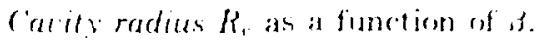

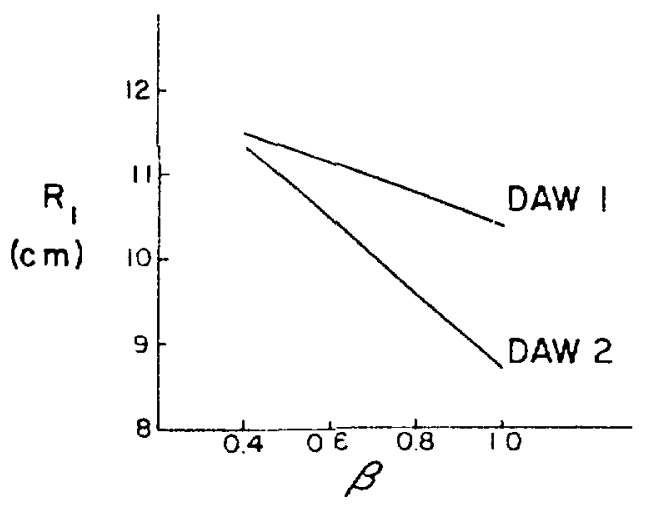

Fig. 15.

Washer radius $R_{1}$ as a function of $\beta$.

\section{RF POWER DISSIPATION IN THE DAW2 CAVITY}

As in the case of DAW1, a major part of the of power fed into the DAW2 cavity is lost in the washer. A typical power-loss distribution is shown in Fig. 18. Figure 19 shows the ratio of power lost in the washer to the total power lost in the whole cavity as a function of $\beta$.

\section{SUMMARY}

High coupling coefficients make configurations DAW1 and DAW2 most attractive for use in high-

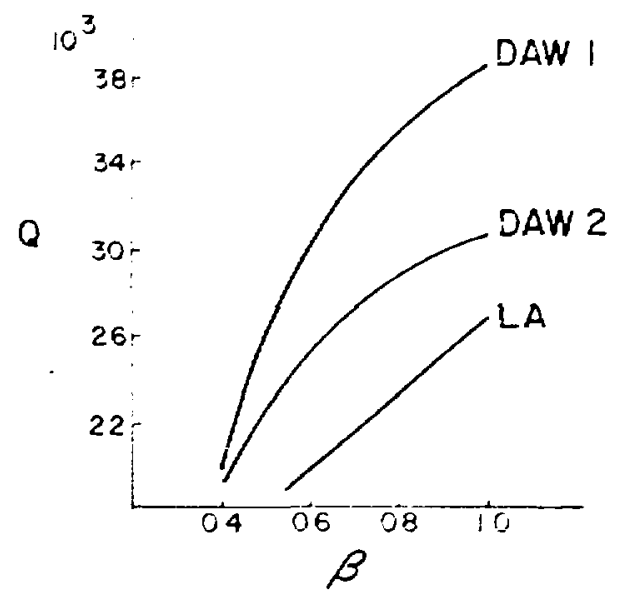

Fis. It

qualien factor le an a function of as.

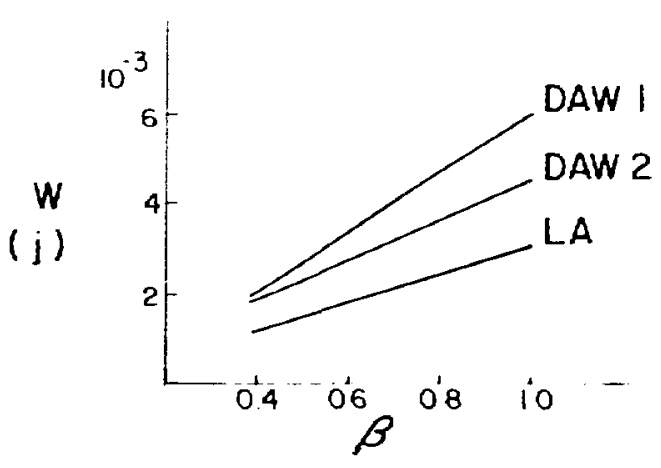

Fig. 17.

Stored energy $W$ in cavity as a function of is.

energy particle accelerators. Both structures are less sensitive to mechanical and alignment errors and to the beam-loading than are conventional coupling structures. Their effective shunt impedances are practically equal and almost the same as the $Z^{2}$ of the $\mathrm{TM}_{01}$-mode cavity. DAW2 has the advantages of a smaller diameter, better field separation of the accelerating $\mathrm{TM}_{02}$-like mode, and a (possibly) higher beam blowup limit. 


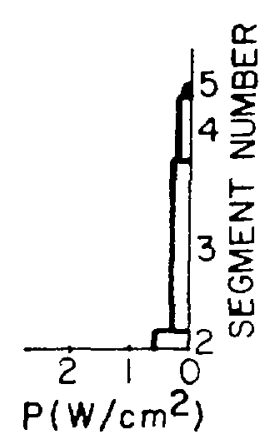

焉

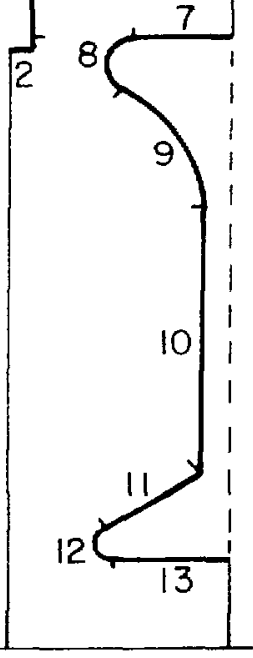

Fig. 18

of pouer loss distrioution on cavity ualls $(\beta=0.6)$.

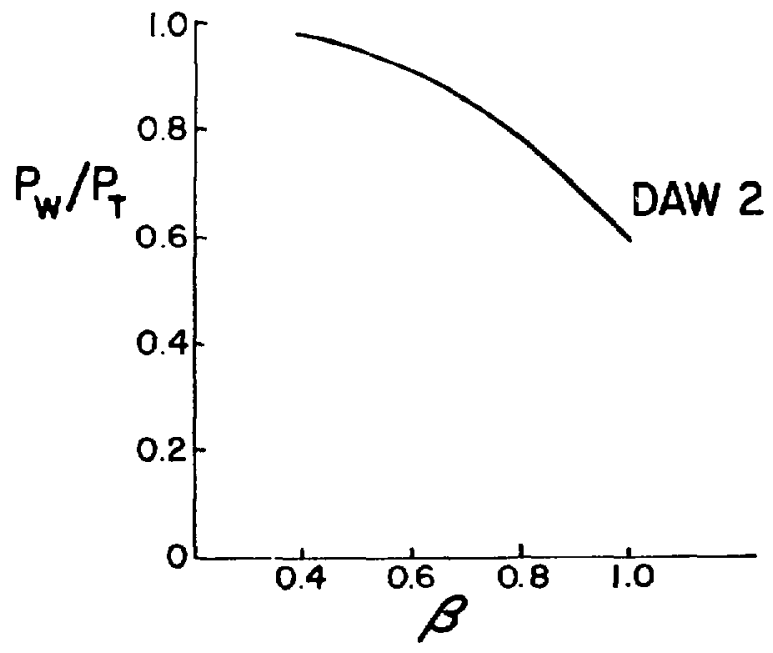

Fig. 19.

Ratio of rf power loss in washer to rf power loss in whole cavity as a function of $\beta$.

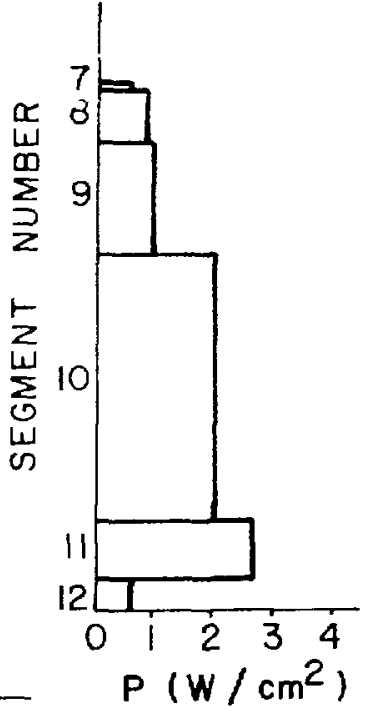

Both DAW1 and DAW2 are axially symmetrical; therefore, their manufacture is relatively simple. Intercavity joints are characterized by small rf current, indicating negligible perturbation from the joint imperfections after final assembly. Larger openings between the neighboring cavities permit high-vacuum conductance. The possibility of washer cooling-liquid transfer outweighs a slight increase of rf power loss in the stems. The use of coaxial couplers for intertank coupling ${ }^{7}$ could result in a reliable and efficient high-energy accelerator.

\section{ACKNOWLEDGMENTS}

We thank D. A. Swenson, R. F. Holsinger, and K. H. Halbach for their many useful discussions and suggestions. We also appreciate the assistance of $P$. Wechsler in running the SUPERFISH program. 


\section{REFLRENCES}

1. V. G. Andrees, "A New Areelerating Structure tur the High-binergy Proton linear Acocteratror Working at $\pi / 2$. Mode," Prese. 2nd All Cinion Particle Arcrelerator conf., Moscow, 1972, p.150 tin Russian).

2. V. (i. Andrees, "Geometry Calculation of a Structure with Sign Alternating Accelerating Field Operating at $\pi / 2$ Mrode," J. Tech. Phys. 41, Txe (IST) (Russian Edd.).

3. V. C. Andrece and V. V. Pashkovskii, "An Ac:celerating Structure with Disks and Drift Tubes for the Proton Linear Accelerator." .J. Tech. Phys. 40. 52:3 (1970) (Russian Fd.).
1. K. H. Halbach and R. F. Hritinger. "STPletelsh A computer lerogram dor Evaluation of RF ravities with (jolindrial Symmetry Part. Arcel. 7, 21:31976).

5. J. J. Manca and E. A. Knapp. TM Mrode Accelerating Cavity Optimizarion." Les Alamos ticien. tific Laboratory report IA-732:; August JaTi.

6. P. M. Lappostrole and A. I. Septier. Fda. Linear A cecelerators 1 Vorth Holland Publi-hinge ('r.. Amsterdam, $197(1)$.

7. .J. J. Manca, "PF ('saxial couplers for High Intensity Linear Acceleraturs." to be published. 